\title{
MAGNET DESIGN FOR THE ISIS SECOND TARGET STATION PROTON BEAM LINE
}

\author{
C.M.Thomas, D.C.Faircloth, S.J.S.Jago, CCLRC, Rutherford Appleton Laboratory, Chilton, \\ Didcot, Oxfordshire, UK.
}

\begin{abstract}
The ISIS facility, based at the Rutherford Appleton Laboratory in the UK, is an intense source of neutrons and muons for condensed matter research. The accelerator facility delivers an $800 \mathrm{MeV}$ proton beam of $2.5 \times 10^{13}$ protons per pulse at $50 \mathrm{~Hz}$ to the present target station. As part of a facility upgrade, it is planned to share the source with a second, $10 \mathrm{~Hz}$, target station. The beam line supplying this target will extract from the existing target station beam line. Electromagnetic Finite Element Modelling techniques have been used to design the magnets required to meet the specified beam line optics. Kicker, septum, dipole, quadrupole, and steering magnets are covered. The magnet design process, involving 2D and 3D modelling, the calculation of ideal shims and chamfers, choice of steel, design of conducting coils, handling of heating issues and eddy current effects, is discussed.
\end{abstract}

\section{SECOND TARGET STATION EPB}

The second target station (TS-2) lies to the south of the ISIS facility. The beam line feeding protons to TS-2 extracts horizontally from the existing Extracted Proton Beam Line (EPB).

\section{BEAM OPTICS}

Knowledge gained from the existing EPB has aided the design of the new beam line. The existing beam line uses $200 \mathrm{~mm}$ nominal aperture components and limits the beam envelope to $75 \%$ occupancy. In order to focus the beam onto the target, the final section of EPB2 requires a larger beam envelope. Therefore it is necessary for the magnets to be of larger core radius to maintain the $75 \%$ occupancy. Thus the aperture is increased to $310 \mathrm{~mm}$.

The transmission efficiency should also be comparable to the $99.9 \%$ achieved on the existing EPB. [1].

\section{MAGNET DESIGN PARAMETERS}

Using the beam optics, the parameters for each magnet can be specified. Primarily, the required field (B) or field gradient $(\mathrm{G})$ within the beampipe, and the magnetic length can be defined. Also characterised at this stage is the required homogeneity of the field within the $75 \%$ occupancy or "good field region". Table 1 shows the magnets planned for EPB2 and their requirements.
Table 1: EPB2 magnets with their required parameters

\begin{tabular}{|l|c|c|c|}
\hline $\begin{array}{c}\text { Magnet } \\
\text { Type }\end{array}$ & $\begin{array}{c}\text { Max. B or } \\
\text { G } \\
\left(\mathrm{T} / \mathrm{Tm}^{-1}\right)\end{array}$ & $\begin{array}{c}\text { Magnetic } \\
\text { Length } \\
(\mathrm{mm})\end{array}$ & $\begin{array}{c}\text { Field } \\
\text { Homogeneity } \\
(\%)\end{array}$ \\
\hline Q11 & $3.8 \mathrm{Tm}^{-1}$ & 500 & \pm 0.5 \\
\hline Q12 & $7.4 \mathrm{Tm}^{-1}$ & 500 & \pm 0.5 \\
\hline Q13 & $8.2 \mathrm{Tm}^{-1}$ & 500 & \pm 0.5 \\
\hline M21 & $0.8 \mathrm{~T}$ & 800 & \pm 0.25 \\
\hline M22 & $1.05 \mathrm{~T}$ & 1250 & \pm 0.25 \\
\hline M23 & $1.58 \mathrm{~T}$ & 1038 & \pm 0.25 \\
\hline M24 & $0.061 \mathrm{~T}$ & 200 & \pm 1 \\
\hline K1 & $0.15 \mathrm{~T}$ & 500 & \pm 0.25 \\
\hline K2 & $0.95 \mathrm{~T}$ & 500 & \pm 0.25 \\
\hline SEPTUM & $1.05 \mathrm{~T}$ & 1458 & \pm 0.25 \\
\hline
\end{tabular}

Q11, Q12 and Q13 are quadrupole magnets used for focussing and defocusing the proton bunches. M21 and M22 are vertical and horizontal dipole magnets used to bend the beam. M23 is a newly designed magnet which will replace an existing magnet within the extraction section where space is very tight. M24 are steering magnets used for slight adjustments to the beam trajectory, and $\mathrm{K} 1$ and $\mathrm{K} 2$ are slow kicker magnets used to deflect 1 in every 5 bunches from the existing EPB towards TS-2.

\section{MAGNET DESIGN PROCESS}

The design process predominantly utilises the 2D and 3D software package OPERA, developed by Vector Fields of Kidlington, England [2]. OPERA is a finite element analysis program for modelling electro-magnetic systems. The EPB2 magnets either provide a linear dipole field or a constant field gradient, so the design process begins with a basic dipole or quadrupole design respectively. Knowledge of ISIS EPB1 magnets and other designs worldwide, combined with basic analytic calculations, help to provide an initial design for each of the magnets described in Table 1. These initial simple designs are then fine-tuned to meet the exact required parameters.

\section{D Modelling}

The two dimensional cross-section of the steel yoke, perpendicular to the beam direction, is drawn in OPERA 2D. Symmetries are utilized to lessen the solving time. In $2 \mathrm{D}$, the quadrupole magnets have 3 -fold symmetry; the dipoles 2-fold. Cross sections through the energising coils are added, with an appropriate current density in $\mathrm{Amm}^{-2}$ 
defined over the whole area. A background region representing the surrounding air must also be added. The finite element mesh is defined at this stage, with higher mesh densities in areas of particular interest such as the beampipe and the steel yoke. Higher mesh densities yield more accurate results.

Figure 1 shows the 2D model of a Q11 magnet, revealing the utilisation of 3-fold symmetry and the finite element mesh.

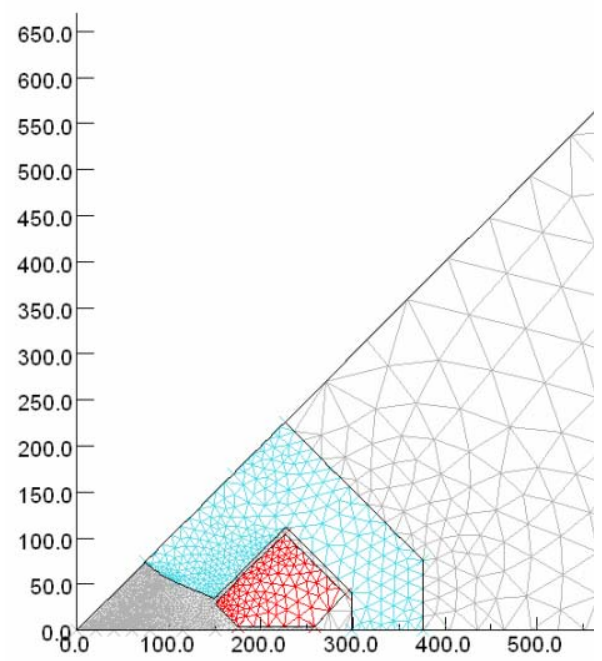

Figure 1: 2D model of Q11 with mesh.

The analysis of the model is then performed using a Newton-Raphson technique. This is an efficient algorithm for finding solutions to real-valued functions; in this case Maxwell's equations. It begins with a value which is close to the root, and through the computation of tangents, an iterative process progresses to a final solution. Having performed the analysis, the magnetic fields are displayed in the post-processor, and the field or field gradient within the good field region checked against the required value (Table 1). The current density within the coil is altered until the required value is reached. The field within the return arms of the yoke must be less than $1.4 \mathrm{~T}$ to limit non-linear saturation effects. If the field it too high, the return arms are widened.

\section{D Modelling}

Having finalised the 2D design by achieving the required $\mathrm{B}$, or $\mathrm{G}$, and homogeneity (see next section) within the good field region, this model is imported into the OPERA 3D pre-processor and extruded in the beam(z-) direction. The symmetries from the $2 \mathrm{D}$ model are once more utilised, with the addition of the z-direction symmetry. Mesh densities are defined for all volumes, with higher densities in volumes of interest such as the beampipe and steel yoke. Mesh density in the background region is kept low to reduce the solving time.

\section{Field Quality}

Table 1 includes the required percentage homogeneity field quality for each magnet. 3D homogeneities of $\pm 0.5 \%$ for the quadrupoles and $\pm 0.25 \%$ for the dipoles have been specified. It is not necessary for the M24 steering magnets, with their low fields, to have such a stringent field quality, so this is set to $\pm 1 \%$. Initially, the $2 \mathrm{D}$ homogeneity is plotted in the OPERA 2D pre-processor as in figure 2.

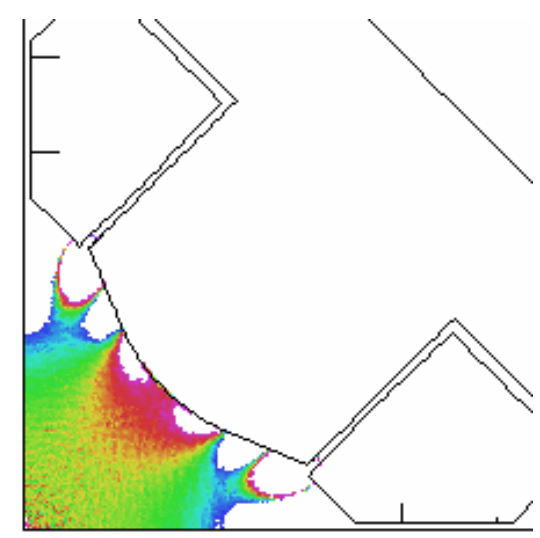

Figure 2: 2D map of $\pm 0.5 \%$ homogeneity for Q11.

3D field quality for each model is calculated using two different methods. The first involves calculating line integrals along the beampipe direction within the good field region. For the quadrupoles, in which field gradient homogeneity is to be $< \pm 0.5 \%$, equation 1 must be satisfied.

$$
\Delta \int\left(\frac{d B_{y}}{d x}\right) d l / \int\left(\frac{d B_{y}}{d x}\right)_{0} d l \leq \pm 5 \times 10^{-3}
$$

The other method uses multipole harmonics. Together with the required dipole or quadrupole harmonics, others are present such as sextupole, octopole etc. which need to be minimised to achieve the necessary field quality. OPERA calculates the values of the multipole harmonics. The values for the unwanted harmonics are normalised and must be less than $1 \times 10^{-4}$. The technique of using multipole harmonics has a direct link to the "rotating coils" method used to test field quality once the magnets have been manufactured.

\section{Shims and Chamfers}

Both quadrupole and dipole magnets require shims to improve the field homogeneity within the good field region of the beampipe. The shims are small pieces of steel added to the ends of the pole faces, which help to shape the field within the required area of beampipe. Experimentation with the use of shims improves the homogeneity in all the magnets. End chamfering is required to compensate for unwanted multipole harmonics introduced by end effects. The side effect of end chamfering is reduced magnetic length. 

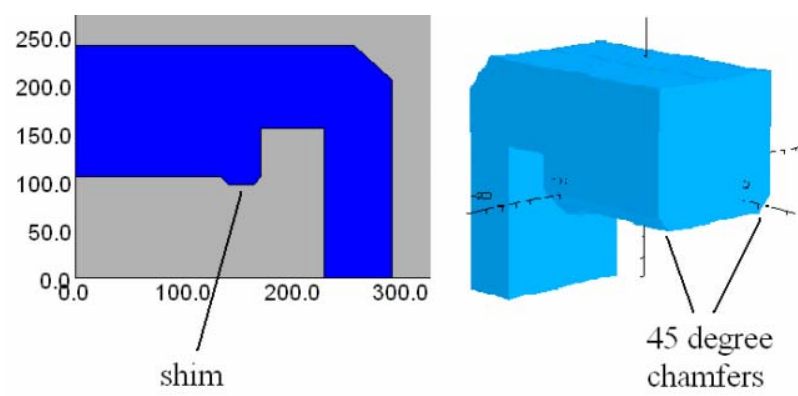

Figure 3: 2D and 3D designs showing shims and chamfers.

\section{COIL DESIGN}

Copper conductors carry current around the steel yoke to induce magnetic fields. Resistance and eddy current losses in the copper result in the production of heat. In order to remove this, water cooling channels approximately 5 to $7 \mathrm{~mm}$ in diameter run through the whole length of conductor. One of the design parameters for each magnet, excluding the septum which requires additional cooling, is such that the temperature difference $\Delta \mathrm{T}$ between the water entering and leaving the magnet will be less than $20^{\circ} \mathrm{C}$. Another design parameter is to keep the pressure difference less than 4 Bar. An approximation to the pressure drop can be gained from equation 2 [3].

$$
\Delta P\left[\mathrm{~kg} / \mathrm{cm}^{2}\right]=5 \times 10^{-5} \mathrm{Lv}^{1.75} / d_{h}^{1.25}
$$

$\mathrm{L}$ is the length of the conductor, $\mathrm{v}$ the velocity of the water flow and $d_{h}$ the hydraulic diameter of the cooling channel. The M24 steering magnets are able to be aircooled due to the low power outputs of the coils.

Coils used in all EPB2 magnets are either of racetrack or bedstead design. Bedstead coils take the ends of the coils further from the magnet yoke, thus improving the field quality, but increasing the complexity and cost of manufacturing. Figure 4 shows drawings of both styles.

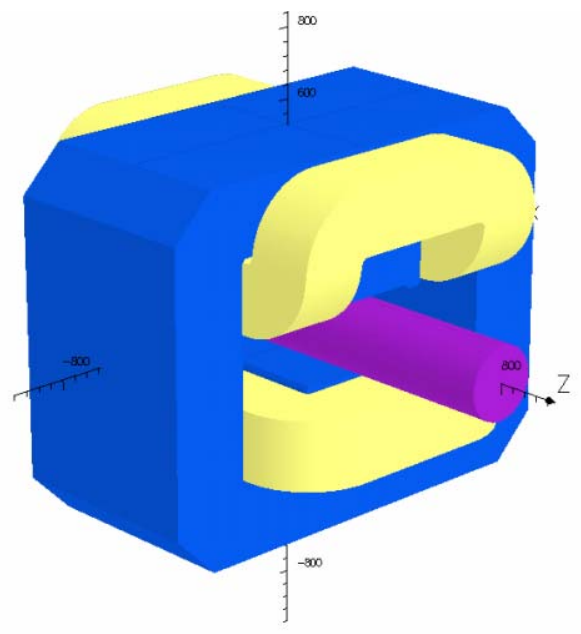

Figure 4: Bedstead (top) and racetrack (bottom) coils.

\section{PULSED DIPOLE “KICKER” DESIGN}

The slow kickers $\mathrm{K} 1$ and $\mathrm{K} 2$ are pulsed dipole magnets deflecting 1 in 5 bunches towards TS-2. They are pulsed at $10 \mathrm{~Hz}$ with each pulse having a $15 \mathrm{~ms}$ rise, approximately $200 \mu$ s flat-top and $15 \mathrm{~ms}$ fall.

\section{Eddy Current Losses}

The pulses induce eddy currents within the conductors and the steel yoke. These circulating currents cause additional losses and heating. To reduce this effect in the yoke, steel laminations of approximately $0.5 \mathrm{~mm}$ thickness are used. This has the effect of restricting the size of the circulating electric currents. In a similar respect: the smaller the cross-sectional area of the conductors the smaller the eddy current losses. However, as the cross-sectional area decreases the resistance losses increase. A compromise must be reached so that the total losses are minimised. The optimum conductors are $5.5 \mathrm{~mm}$ square, with $2.5 \mathrm{~mm}$ diameter cooling channel.

\section{Remanent Field}

The kickers are pulsed such that they provide a bending field for one in every 5 bunches. It is essential that the remaining 4 bunches pass straight though the magnets, experiencing very minimal deflection. The deflection for these bunches must be less than $1 \mathrm{mrad}$. The remanent field of the larger kicker magnet was estimated (using Eq.3) to be $0.64 \mathrm{mT}$. This equates to a deflection of $0.05 \mathrm{mrad}$ which is acceptable.

$$
B_{r}=\frac{-\mu_{0} H_{c} L}{g}
$$

$B_{r}$ represents the remanent field, $H_{c}$ the coercivity of the steel, $\mathrm{L}$ the flux path length and $\mathrm{g}$ the gap between poles. Special grades of steel are available which can lower the remanent field further if necessary.

\section{SEPTUM DESIGN}

The ISIS EPB2 septum will be DC powered. The magnet requires a large current of $9000 \mathrm{~A}$ to produce the $1.05 \mathrm{~T}$ required, and also a large amount of water cooling. The straight through part of the septum is made from mild steel which shields this section from the magnetic field. There are two coils on the magnet, each with 7 turns. The conductors are rectangular with $\sim 250 \mathrm{~mm}^{2}$ cross-sectional area, and incorporate a cooling channel $5.5 \mathrm{~mm}$ in diameter. Calculations have shown that a flow rate of $300 \mathrm{l} / \mathrm{min}$ will allow the temperature change to be controlled to 25 degrees and the pressure drop to 3.5 Bar.

\section{REFERENCES}

[1] "Proton Beam Line for the ISIS Second Target Station", D.Adams, EPAC 2004, TUPLT136

[2] Vector Fields, OPERA Version 10

[3] "A Selection of Formulae and Data Useful for the Design of A.G.Synchrotrons", MPS-SI/Int. DL/68-3, CERN (1968). 